\title{
Avaliação da Criatividade a Partir do Controle do Nível de Inteligência em uma Amostra de Crianças
}

\author{
Tatiana de Cássia Nakano ${ }^{1}$ \\ Departamento de Pós-Graduação em Psicologia da Pontifícia Universidade Católica \\ de Campinas, Campinas, Brasil \\ Maíra Esteves Brito \\ Faculdade de Ciências Médicas da Universidade Estadual de Campinas, Campinas, Brasil
}

\begin{abstract}
Resumo
Buscando enriquecer a discussão acerca da relação entre criatividade e inteligência, uma amostra composta por 135 crianças (média de 10,3 anos, $D P=1,05$ ), estudantes de $4^{\circ}$ ao $6^{\circ}$ ano do Ensino Fundamental, respondeu à Bateria de Provas de Raciocínio Infantil e ao Teste de Criatividade Figural Infantil. Duas análises foram realizadas a partir de critérios diferentes de controle do nível de inteligência dos participantes (alta, média e baixa inteligência). Quando utilizado o critério da média e desvio padrão (abaixo de $-1 D P$, entre $-1 D P$ e $+1 D P$ e acima de $+1 D P$ ), os resultados da Análise da Variância indicaram a interação entre as variáveis sexo e grupo como marginalmente significativa no fator 2 (emocional), entretanto, quando uma nova divisão é realizada a partir do percentil (abaixo do P25, média e acima do P75), a variável grupo passa a influenciar significativamente o resultado dos participantes no Fator 1 da criatividade (Enriquecimento de Idéias). O estudo ainda apontou correlação baixa entre os dois construtos $(r=0,22)$.
\end{abstract}

Palavras-chave: Desenho, raciocínio, infantil.

\section{Creativity Assessment: Control of the Level of Intelligence in a Sample of Children}

\begin{abstract}
With the aim of enrich the discussion about the relationship between creativity and intelligence, a sample of 135 children (mean 10.3 years, $S D=1.05$ ), students from fourth to sixth year of elementary school, answered the battery of reasoning tests Children and the Child Figural Creativity Test. Two different tests were conducted using different criteria of dividing the participants based on the control of intelligence level (high, medium and low intelligence). When this division was based in the mean and standard deviation ( $1 S D$ below-between- $1 S D$ and $1 S D+$ and above $+1 S D)$, the results of analysis of variance showed the interaction between gender and group like marginally significant in factor 2 (emotional), however, when a new division is done from the percentile (below the P25, P75 and above the average), the variable group is to influence significantly the result in factor 1 (Enrichment of Ideas). The study also showed low correlation between the constructs $(r=.22)$.
\end{abstract}

Keywords: Drawing, reasoning, children.

Endereço para correspondência: Departamento de Pós-Graduação em Psicologia, Pontifícia Universidade Católica-Campinas, Av. John Boyd Dunlop, s/n, Jardim Ipaussurama, Campinas, SP, Brasil 13060-904. E-mail: tatiananakano@hotmail.com 


\section{La Evaluación de la Creatividad a Partir de Control de Nivel de Inteligencia en una Muestra de Niños}

\section{Resumen}

Buscando enriquecer el debate sobre la relación entre la creatividad y la inteligencia, una muestra de 135 niños (media de 10.3 años, $S D=1.05$ ), estudiantes de cuarto a sexto grado de la escuela primaria, respondió a la Batería del Razonamiento para Niño y la Prueba de Creatividad Figurativo para Niño. Dos análisis se realizaron con criterios diferentes para control de la inteligencia de los participantes (inteligencia alta, media y baja). Cuando los participants se dividieron en tres grupos con el criterio de la media y la desviación estándar (abajo de $1 S D$; entre $-1 S D$ y $+1 S D$; encima de $+1 S D$ ), los resultados del análisis de varianza indica la interacción entre las variables género y grupo como marginalmente significativo en el factor 2 (emocional), sin embargo, cuando una nueva división se realiza desde el percentil (debajo del P25, media y por encima del P75), la variable grupo comienza a influir significativamente en los resultados de los participantes en el factor 1 de creatividad (enriquecimiento de ideas). El estudio también mostró una baja correlación entre las dos construcciones $(r=.22)$.

Palabras clave: Diseño, razonamiento, niño.

Pesquisadores interessados no estudo da criatividade têm se concentrado em responder cinco questões, de acordo com Becker (1995): O que é criatividade? Quem é criativo? Quais são as características das pessoas criativas? Quem pode beneficiar-se da criatividade? Pode a criatividade ser aumentada por meio de esforços conscientes? Não há dúvidas que estas constituem-se em importantes questões para a compreensão da criatividade, embora haja um outro tema que tem gerado um grande interesse por parte da comunidade científica e que não foi apontado pelo autor: a relação entre criatividade e inteligência.

Pesquisadores vêm, há um longo tempo, debatendo sobre a natureza da relação entre os dois construtos. Mesmo após um grande número de pesquisas desenvolvidas, o consenso está longe de ser atingido, de forma que, apesar de ser um tema sendo bastante estudado, as investigações atuais seguem questionando a existência dessa relação e o nível da associação, além da sua estabilidade no tempo e possibilidade de generalização para populações distintas (Elisondo \& Donolo, 2010). O interesse na temática teve início por volta do ano de 1900, ocasião em que os pesquisadores começaram a medir as diferenças individuais em inteligência, principalmente por meio dos testes de Binet, os quais incluíam itens que os pesquisadores acreditavam requerer imaginação e o que eles chamaram de pensamento divergente (Albert \& Runco, 1999).

Desde então, resultados de pesquisas empíricas têm sugerido a existência de pelo menos alguma relação entre criatividade e inteligência, conforme apontado por Kim (2005) que, após a realização de meta-análise de 21 estudos que continham 447 coeficientes de correlação e mais de quarenta e cinco mil participantes, afirmou a existência da relação, pequena $(r=0,17)$ mas positiva entre os dois construtos. O questionamento aos resultados encontrados na literatura amparam-se na constatação de que o valor e a significância dessa relação irá depender do tipo de criatividade e de inteligência que são medidas, de forma que diferentes resultados são relatados de acordo com o tipo de estudo, sendo que, os achados, aparentemente contraditórios, podem ser explicados, em parte, pela heterogeneidade das medidas empregadas e tipo de população estudada.

Nesse sentido, pode-se encontrar três vertentes de pesquisadores. Na primeira delas, os autores afirmam a existência de relação entre criatividade e inteligência (Nogueira \& Pereira, 2008), de forma que a realização criadora poderia requerer criatividade e inteligência conjuntamente (Sternberg, 2001). Para os teóricos 
adeptos dessa linha de pensamento, os construtos poderiam ser considerados distintos, embora relacionados (Preckel, Holling, \& Wiese, 2006; Sternberg, 2000) de forma que um nível moderado de inteligência parece ser necessário para o aparecimento da criatividade (Kneller, 1971).

Uma segunda visão defende a idéia de que criatividade e inteligência seriam dois construtos completamente diferentes e independentes (Getzels \& Jackson, 1962; Hattie \& Rogers, 1986) visto que, uma pessoa com alta inteligência poderia, ou não, ser altamente criativa, de forma a se afirmar que os dois construtos podem ser separados conceitualmente (Kim, Cramond, \& Bandalos, 2006; Sternberg, 1984). Assim, o QI por si só não é visto como único fator para a determinação do talento criativo (Sternberg \& O'Hara, 2000), uma vez que a criatividade pode ser vista como construção suficientemente distinta da inteligência (Rindermann \& Neubauer, 2004), confirmada pela baixa correlação encontrada entre os testes de inteligência e criatividade (Edwards \& Tyler, 1965; Russo, 2004; Wechsler \& Richmond, 1984).

A terceira vertente defende a idéia de que a relação pode não ser simplesmente linear, mas existente a partir de um certo nível de inteligência (teoria do threshold), (Mouchiroud \& Lubart, 2002). Para os autores dessa corrente de pensamento, os dois construtos estariam correlacionados a partir de um certo ponto do corte, mais comumente um valor de QI em torno de 120 (Kneller, 1971; Lubart, 2007; Preckel et al., 2006; Shaffer, 2005), de forma que valores iguais ou superiores a este facilitariam a entrada em áreas nas quais um trabalho altamente criativo é possível, embora, na opinião dos autores, a partir de um certo nível de QI não haveria mais benefício suplementar, de forma que, abaixo desse valor a correlação não seria significativa.

Destaque deve ser dado ao fato de que a literatura científica ainda é bastante restrita quando se considera a investigação da relação entre os construtos em crianças pequenas ou pessoas mais velhas, uma vez que a maior parte dos estudos tem como foco a investigação de jovens adultos (Aguirre \& Conners, 2010). Vê-se que essa lacuna não se diferencia muito da situação encontrada em relação à avaliação isolada da criatividade. Diversos levantamentos têm indicado que a população de adultos tem sido a mais estudada nas pesquisas, vindo, a seguir, as crianças (Alencar, 1997; Wechsler \& Nakano, 2003; Zanella \& Titon, 2005), sendo praticamente inexistente os estudos enfocando a criatividade na velhice (Wechsler \& Nakano, 2002). Esses resultados sugerem:

a importância da realização de diversos estudos sobre a criatividade em faixas etárias diversificadas, incluindo crianças bem pequenas até adultos, passando por várias idades e níveis de escolaridade, de forma que possa ser mais delineado o percurso da criatividade nas diversas épocas do desenvolvimento humano. (Wechsler \& Nakano, 2006, p. 216)

$\mathrm{O}$ estímulo a pesquisas que tenham como foco a criatividade de crianças torna-se importante diante da constatação de que estudar a criatividade, aptidão fluída e de difícil mensuração, constitui-se em uma tarefa ainda mais difícil visto que nessa faixa etária existe uma criatividade espontânea (criatividade primária) e ainda não a criatividade controlada e disciplinada (criatividade secundária), que caracteriza a adultez (Nogueira \& Pereira, 2008). Neste sentido Pereira (2000) defende que a criatividade é uma aptidão demasiadamente instável na infância, devido às produções precárias (o que reflete, por exemplo, na baixa estabilidade temporal dos resultados) e de difícil mensuração (visto que os instrumentos de avaliação da criatividade possuem baixos coeficientes de validade devido às mudanças muito rápidas nessa fase).

Em relação à criatividade infantil, Martinez e Lozano (2010) argumentam que nos primeiros anos, aprendizagem e criatividade estão ligados ao desenvolvimento pessoal e à medida em que a idade da criança aumenta, o ato criativo se exterioriza, até o ponto que pode separar-se desse desenvolvimento, quando a criatividade deixaria de ser tão "evolutiva" para transformar-se em mais "objetal" (através das produções, realizações e capacidades). Estudos voltados à essa faixa etária também podem contribuir no sentido de desfazer os mitos associados à criatividade, 
tal como o de que as crianças são mais criativas que os adultos, conforme apontado por Sawyer (2006). Segundo o autor, muitos acreditam que as crianças são naturalmente criativas, diferentemente dos adultos, os quais seriam influenciados pela pressão pela adequação social imposta pela sociedade, escola e família (as quais atuariam como repressoras desse impulso criativo natural). Assim, Aguirre e Conners (2010) sugerem que pode haver diferenças bastante importantes de serem consideradas quando se comparam crianças com alta inteligência e aquelas com alta criatividade, fato que precisa ser reconhecido quando se trabalha com essa faixa etária, de maneira a considerar a necessidade de promoção das duas características, especialmente em programas voltados ao atendimento de crianças superdotadas e talentosas.

Diante do destaque que as discussões acerca da relação entre criatividade e inteligência vêm recebendo no meio científico, da importância da investigação da criatividade infantil e da constatação da existência de lacunas no estudo dessa relação em crianças, o presente estudo visou realizar a avaliação da criatividade a partir do controle do nível de inteligência em uma amostra de crianças, verificando ainda a influência das variáveis sexo e nível de inteligência no desempenho criativo.

\section{Método}

\section{Participantes}

A amostra foi composta por 135 crianças com idades entre 8 e 14 anos (média de 10,3, $D P=1,05$ ), sendo 69 do sexo feminino e 66 do masculino, selecionadas dentro de uma amostra de conveniência. Os participantes eram estudantes de quarto $(n=42)$, quinto $(n=30)$ e sexto ano $(n=63)$ do Ensino Fundamental, provenientes de uma escola pública situada no interior do Estado de São Paulo.

\section{Instrumentos}

Teste de Criatividade Figural Infantil (Nakano, Wechsler, \& Primi, 2011). Instrumento no qual os examinandos são incentivados a comporem desenhos em três atividades a partir de estímulos pouco definidos. As características avaliadas são 12: Fluência (número de respostas adequadas), Flexibilidade (diversidade de tipos ou categorias de idéias), Elaboração (adição de detalhes ao desenho básico), Originalidade (idéias incomuns), Expressão de Emoção (expressão de sentimentos, tanto nos desenhos quanto nos títulos), Fantasia (presença de seres imaginários, de contos de fada ou ficção científica), Movimento (clara expressão de movimento nos desenhos ou títulos), Perspectiva Incomum (pessoas ou objetos desenhados sobre ângulos não usuais), Perspectiva Interna (visão interna de objetos ou parte do corpo das pessoas, sob a forma de transparência), Uso de Contexto (criação de um ambiente para o desenho), Extensão de Limites (estender os estímulos antes de concluir os desenhos), Títulos Expressivos (ir além da descrição óbvia do desenho, abstraindo-o).

A correção do instrumento permite a obtenção de pontuações totais para cada uma das características, considerando-se a atividade de ocorrência, somadas de forma a constituir quatro fatores da criatividade (Nakano \& Primi, 2012): Fator Enriquecimento de Idéias (capacidade de ver a situação de uma forma mais detalhada, por um ponto de vista diferente); Fator Emotividade (composto por características que envolvem o uso de recursos criativos ligados à uma percepção mais emocional); Fator Preparação Criativa (separa basicamente algumas características avaliadas na primeira atividade do teste, funcionando como um esquentamento para a realização das demais atividades); Fator Aspectos Cognitivos (composto por características criativas que fazem uso de recursos cognitivos, que envolvem a busca de soluções diferenciadas, originais e que vão além dos limites estabelecidos), além de uma pontuação total no instrumento.

Estudos visando a busca por evidências de validade e precisão do instrumento indicaram valores entre 0,81 e 0,94 de correlação para validade concorrente com o Teste Figural de Torrance e índices entre 0,84 e 0,95 de correlação para a precisão por meio do teste e reteste (Nakano \& Wechsler, 2006). Os quatro fatores apresentaram bom ajuste ao modelo, verificado 
por meio de estudos envolvendo uso da Teoria de Resposta ao Item (Nakano et al., 2011), de maneira que os resultados das pesquisas apontam bons indicadores de precisão e validade em amostras brasileiras.

Bateria de Provas de Raciocínio Infantil (Primi \& Almeida, n.d.). Instrumento que avalia habilidades cognitivas (inteligência fluida e cristalizada) por meio de quatro subtestes: raciocínio abstrato (RA), que avalia basicamente a Inteligência Fluida (Gf), definida como a capacidade de raciocinar em situações novas, criar conceitos e compreender implicações; raciocínio verbal $(\mathrm{RV})$, que avalia tanto a Inteligência Fluida (Gf), quanto a Cristalizada (Gc), definida por sua vez, como a extensão e profundidade do conhecimento verbal vocabular e a capacidade de raciocinar utilizando conceitos previamente aprendidos; raciocínio numérico $(\mathrm{RN})$, que mede a Inteligência Fluida (Gf) e a Habilidade Quantitativa $(\mathrm{Gq})$, definida como a compreensão de conceitos quantitativos básicos, como soma, subtração, multiplicação e divisão e manipulação de símbolos numéricos; e raciocínio prático (RP), que avalia a Inteligência Fluida (Gf) por meio de problemas do dia a dia.

Importante salientar que, no momento, somente a versão para adultos encontra-se publicada e autorizada para uso no Brasil. A versão infantil utilizada no presente estudo, encontra-se em processo de coleta de dados e ampliação da amostra para posterior publicação. Entretanto seus estudos psicométricos já evidenciaram a validade e precisão da mesma para identificação da inteligência em crianças e adolescentes, através de estudos conduzidos por Cruz (2008) junto a 289 alunos de $1^{\text {a }}$ até $5^{\text {a }}$ série do Ensino Fundamental. Os estudantes foram submetidos à aplicação da BPR-5i, Matrizes Progressivas Coloridas de Raven, Teste de Competência de Leitura Silenciosa, Teste de Compreensão de Sentenças Escritas e um Questionário de Identificação Pessoal. Os coeficientes de consistência interna variaram de 0,79 a 0,94 e os de precisão pelo método teste-reteste de 0,63 a 0,74 . Análises demonstraram a validade convergente da bateria com o Raven (com correlações variando entre 0,38 e 0,69$)$, assim como validade de critério com notas escolares $(0,59)$.

\section{Procedimentos}

Inicialmente a pesquisa foi submetida à apreciação do Comitê de Ética em Pesquisa da Pontifícia Universidade Católica de Campinas (PUC-Campinas), tendo sido aprovada sob número 850/09. Após esse processo, primeiramente foi realizado um contato com a direção da escola para que fossem explicitados os objetivos da pesquisa e para obtenção da autorização necessária. Após a consecução da devida autorização, foram escolhidas aleatoriamente duas salas de cada ano escolar envolvido, ou seja, quarto, quinto e sexto anos.

Os professores foram contatados para que em dia e horário marcado os instrumentos fossem aplicados de forma a não prejudicar o andamento das atividades acadêmicas. Por se tratarem de crianças, foi enviado previamente aos pais o Termo de Consentimento Livre e Esclarecido. Os instrumentos foram aplicados de forma coletiva em sala de aula, tendo-se alterado a ordem de aplicação dos instrumentos.

Os testes foram corrigidos de acordo com os manuais de forma que o instrumento de raciocínio deu origem a cinco pontuações (RA, $\mathrm{RV}, \mathrm{RP}, \mathrm{RN}$ e raciocínio total) e o instrumento de criatividade também a cinco pontuações (Fator 1, Fator 2, Fator 3, Fator 4 e criatividade total).

A partir da estimativa da média $(X=48,83)$ e desvio padrão $(D P=17,29)$ do resultado total da BPRi os participantes foram divididos em três grupos: alta inteligência (aqueles que obtiveram resultado total acima de 1 desvio padrão), média inteligência (aqueles cujos resultados totais situaram-se entre $-1 D P$ e $+1 D P$ ) e baixa inteligência (aqueles cujos resultados totais estiveram abaixo de 1 desvio padrão). Assim o grupo com alta inteligência ficou composto por 22 crianças, o grupo de baixa inteligência por 23 crianças e outras 90 crianças compuseram o grupo intitulado média inteligência.

A correlação de Pearson foi empregada com a finalidade de se verificar a relação entre as medidas dos dois instrumentos e a Análise Univariada da Variância buscou investigar a influência das variáveis sexo e grupo no desempenho dos participantes. 
Tabela 1

Médias e Desvios Padrão por Nível de Inteligência e Sexo no TCFI e BPRI

\begin{tabular}{|c|c|c|c|c|c|c|c|c|c|c|}
\hline Grupo & & & $\mathrm{RV}$ & $\mathrm{RP}$ & RA & $\mathrm{RN}$ & F1 & $\mathrm{F} 2$ & F3 & F4 \\
\hline \multirow{4}{*}{$\begin{array}{c}\text { Média } \\
\text { inteligência }\end{array}$} & \multirow{2}{*}{ Fem. } & Média & 17,96 & 7,21 & 14,53 & 9,83 & 53,96 & 0,98 & 4,72 & 41,77 \\
\hline & & $D P$ & 4,30 & 3,03 & 4,82 & 6,76 & 21,98 & 1,80 & 3,37 & 14,20 \\
\hline & \multirow{2}{*}{ Masc. } & Média & 17,98 & 5,16 & 15,35 & 11,72 & 55,98 & 1,88 & 5,30 & 40,40 \\
\hline & & $D P$ & 4,25 & 3,12 & 5,52 & 6,55 & 25,62 & 3,26 & 3,70 & 14,91 \\
\hline \multirow{4}{*}{$\begin{array}{c}\text { Alta } \\
\text { inteligência }\end{array}$} & \multirow{2}{*}{ Fem. } & Média & 21,83 & 9,00 & 21,92 & 19,25 & 69,83 & 3,50 & 8,00 & 44,92 \\
\hline & & $D P$ & 3,63 & 1,70 & 1,62 & 3,16 & 32,17 & 6,84 & 6,88 & 6,69 \\
\hline & \multirow{2}{*}{ Masc. } & Média & 21,90 & 10,50 & 21,70 & 19,80 & 64,00 & 1,10 & 5,40 & 45,90 \\
\hline & & $D P$ & 2,37 & 1,84 & 2,40 & 2,57 & 28,35 & 1,28 & 3,97 & 17,61 \\
\hline \multirow{4}{*}{$\begin{array}{c}\text { Baixa } \\
\text { inteligência }\end{array}$} & \multirow{2}{*}{ Fem. } & Média & 11,10 & 4,40 & 6,10 & 0,50 & 57,80 & 1,60 & 5,80 & 39,00 \\
\hline & & $D P$ & 5,30 & 2,11 & 4,95 & 0,85 & 34,41 & 2,59 & 4,15 & 23,02 \\
\hline & \multirow{2}{*}{ Masc. } & Média & 9,85 & 3,69 & 6,69 & 1,38 & 50,92 & 0,69 & 5,85 & 35,08 \\
\hline & & $D P$ & 6,02 & 2,95 & 5,76 & 3,66 & 34,51 & 1,31 & 4,89 & 15,85 \\
\hline \multirow{4}{*}{$\begin{array}{l}\text { Amostra } \\
\text { Total }\end{array}$} & \multirow{2}{*}{ Fem. } & Média & 17,64 & 7,12 & 14,59 & 10,12 & 57,28 & 1,51 & 5,45 & 41,91 \\
\hline & & $D P$ & 5,28 & 3,00 & 6,28 & 7,81 & 26,20 & 3,39 & 4,38 & 14,72 \\
\hline & \multirow{2}{*}{ Masc. } & Média & 16,97 & 5,68 & 14,61 & 10,91 & 56,20 & 1,53 & 5,42 & 40,18 \\
\hline & & $D P$ & 5,80 & 3,59 & 6,87 & 7,87 & 27,76 & 2,77 & 3,93 & 15,59 \\
\hline
\end{tabular}

\section{Resultados e Discussão}

Inicialmente são apresentadas as médias e desvios padrão para cada um dos grupos, nos dois instrumentos, classificadas de acordo com o sexo do participante.

A simples observação das médias, a partir dos resultados da Tabela 1, indicam oscilação de desempenho em função do sexo quando se analisa a amostra total e os grupos separadamente. $\mathrm{Na}$ amostra total há, de uma forma geral, um melhor desempenho dos participantes do sexo feminino que obtêm maiores médias nas provas de raciocínio verbal, raciocínio prático, fator 1 e fator 4 da criatividade. Os homens destacam-se somente no raciocínio numérico. Os desempenhos se assemelham nas provas de raciocínio abstrato, além do fator 2 e 3 da criatividade. Entretanto quando se controla o nível de inteligência e se analisam os grupos separadamente, veremos que os resultados se alteram.
No grupo com média inteligência vemos uma inversão, de forma que os homens apresentam maiores médias na maior parte das medidas (RA, RN, F1, F2 e F3). Nesse grupo as mulheres destacam-se no RP e F4, sendo o desempenho bastante semelhante entre os sexos em relação à prova de RP.

No grupo com baixa inteligência nota-se melhores resultados são obtidos pelas mulheres (RV, RP, F1, F2, F4). Homens destacam-se em RA e RN, sendo o resultado semelhante entre os sexos no F3 da criatividade. No grupo com alta inteligência nota-se um equilíbrio maior entre os sexos, sendo que as mulheres destacam-se em RA, F1, F2 e F3, ao passo que os homens em RP, $\mathrm{RN}$ e F4. Desempenho semelhante se dá na prova RV. O que se pode notar é que, independente do grupo, os homens destacam-se em raciocínio numérico e as mulheres, de uma forma geral, nas medidas de criatividade (com exceção do grupo de inteligência média). 
Esses resultados confirmam os dados relatados nos manuais dos respectivos instrumentos, ao confirmar na BPR-5 a superioridade masculina nos testes de desempenho numérico (Almeida \& Primi, 2000; Cruz, 2008). De uma forma geral, segundo Almeida, Lemos e Primi (2011), os resultados na bateria apontam para desempenhos superiores por parte do sexo masculino. "Estas diferenças são, no entanto, bastante circunscritas às provas de raciocínio especial, numérico e mecânico, onde as mesmas diferenças apresentam, ao longo dos tempos e dos vários estudos, significância estatística" (p. 302).

Convém resssaltar, entretanto, que diversas pesquisas (Almeida, 1988; Almeida \& Campos, 1985; Bornstein, 1984; Camarata \& Woodcock, 2006; Jensen, 1998; Lemos et al., 2010; Reynolds, Keith, Ridley, \& Patel, 2008) têm demonstrado que nas provas que envolvem conteúdo verbal e abstrato essa superioridade por parte dos homens não existe ou não sua significância não é tão frequente, de modo a se afirmar a igualdade dos gêneros. Há ainda, na literatura, estudos nos quais as mulheres superam os homens na prova de raciocínio verbal (Ackerman, Bowen, Beier, \& Kanfer, 2001; Back \& Dana, 1977; Halari et al., 2005; Nisbett et al., 2012) e resultados contrários (Hamilton \& McKenna, 1965; Keith, Reynolds, Patel, \& Ridley, 2008), de modo que recomenda-se cautela na interpretação das diferenças relatadas no presente estudo. Isso porque a questão da diferença de gênero nas provas de habilidade cognitiva ainda tem se mostrado controversa na literatura científica (Doherty, Kovas, \& Plomin, 2011; Kimura, 1987; van der Sluis et al., 2008), de modo que, se tais diferenças existem, ainda não se encontra claramente definido como elas emergem e se desenvolvem (Kramer, Kaplan, Delis, O’Donnell, \& Prifitera, 1997).

Da mesma maneira são encontrados estudos que apontam a superioridade feminina em relação à criatividade (Alencar, 1975; Dudek, Strobel, \& Runko, 1993; Osborn, 1975; Rodrigues \& Alencar, 1983; Virgolim \& Alencar, 1993; Wechsler, 1987), segundo os quais, de modo geral, o grupo feminino, geralmente, tende a apresentar escores superiores quando comparado ao masculino, na maior parte das medidas de criatividade. Entretanto, assim como destacado em relação à inteligência, a questão das diferenças de gênero em criatividade também não se encontram definidas de forma consensual, sendo relatados resultados que se agrupam em quatro posicionamentos: o primeiro afirmando a superioridade do sexo feminino, o segundo defendendo a superioridade do sexo masculino, o terceiro afirmando a não existência de diferenças e o quarto posicionamento que aponta para uma oscilação de desempenho, dependendo do conteúdo avaliado (Reese, Lee, Cohen, \& Puckett, 2001).

Como consequência, quando se procuram na literatura científica, respostas acerca da existência de diferença na criatividade devido ao gênero, o que se encontra é que esta tem se mostrado uma questão polêmica. Segundo Baer (1999), questões relativas ao como e o porquê homens e mulheres diferem no pensamento criativo, sempre foram elaboradas e diversos estudos tentaram respondê-las, sendo que os resultados mostraram-se completamente confusos e contraditórios. Desse modo, cautela é recomendada na interpretação dos resultados aqui apresentados, restringindo os mesmos à amostra envolvida e à medida utilizada.

Assim, diante das aparentes diferenças, e considerando-se que os grupos foram classificados de acordo com o desempenho total no teste de inteligência, a influência dessa variável foi calculada a partir da Análise Multivariada da Variância para os quatro fatores de criatividade, cujos resultados encontram-se na Tabela 2.

Os resultados da Análise da Variância indicaram que o desempenho nos quatro fatores da criatividade não se mostrou influenciado pelo nível de inteligência dos participantes (grupo) e nem pelo sexo do participante. Somente a interação entre sexo e grupo no fator 2 (emocional) mostrou-se marginalmente significativa, a favor das mulheres.

Os resultados levantaram uma hipótese acerca do motivo pelo qual as diferenças não foram significativas. Talvez o método utilizado para divisão dos grupos (de acordo com o critério de $D P$ ) tenha acabado por criar grupos muito extremos de baixa e alta inteligência, centrando 
Tabela 2

Análise Univariada da Variância para Grupo, Sexo e Interação no Instrumento de Criatividade

\begin{tabular}{ccrrrrc}
\hline Variável & Variável dependente & Soma dos quadrados & $g l$ & Médias ao quadrado & \multicolumn{1}{c}{$F$} & \multicolumn{1}{c}{ Sig. } \\
\hline Grupo & F1 & 2669,212 & 2 & 1334,606 & 1,843 & 0,162 \\
& F2 & 17,139 & 2 & 8,570 & 0,915 & 0,403 \\
& F3 & 54,307 & 2 & 27,154 & 1,588 & 0,208 \\
Sexo & F4 & 777,971 & 2 & 388,985 & 1,690 & 0,189 \\
& F1 & 282,361 & 1 & 282,361 & 0,390 & 0,533 \\
& F2 & 14,262 & 1 & 14,262 & 1,522 & 0,220 \\
& F3 & 9,635 & 1 & 9,635 &, 563 & 0,454 \\
Grupo * Sexo & F4 & 45,898 & 1 & 45,898 &, 199 & 0,656 \\
& F1 & 525,336 & 2 & 262,668 & 0,363 & 0,696 \\
& F2 & 54,335 & 2 & 27,168 & 2,900 & 0,059 \\
& F3 & 44,385 & 2 & 22,193 & 1,298 & 0,277 \\
& F4 & 66,973 & 2 & 33,487 & 0,145 & 0,865 \\
\hline
\end{tabular}

a maior parte dos participantes em um grupo médio, conforme esperado, dada a distribuição normal da amostra. Entretanto, a fim de testar se os resultados se manteriam, uma nova divisão dos grupos foi realizada considerando-se um segundo critério de divisão: o percentil. Dessa forma novamente três grupos foram criados: inteligência abaixo da média (resultados igual ou abaixo de 42 pontos no total do teste de inteligência, correspondendo ao percentil entre 1 e 33, em um total de 48 participantes), média inteligência (resultados entre 43 e 57 no teste de inteligência, correspondentes ao percentil entre 34 e 66, em um total de 42 participantes) e inteligência acima da média (resultados maiores que 58 pontos no teste de inteligência, correspondente ao percentil entre 67 e 99 , em um total de 45 participantes) de forma que, a partir desse método o

Tabela 3

Análise Univariada da Variância para Grupo, Sexo e Interação

\begin{tabular}{crrrrrc}
\hline Variável & Variável dependente & Soma dos quadrados & $g l$ & Médias ao quadrado & \multicolumn{1}{c}{$F$} & Sig. \\
\hline Grupo & F1 & 6632,841 & 2 & 3316,421 & 4,780 & 0,010 \\
& F2 & 41,574 & 2 & 20,787 & 2,175 & 0,118 \\
& F3 & 85,361 & 2 & 42,681 & 2,535 & 0,083 \\
Sexo & F4 & 548,391 & 2 & 274,196 & 1,179 & 0,311 \\
& F1 & 0,598 & 1 & 0,598 & 0,001 & 0,977 \\
& F2 & 0,312 & 1 & 0,312 & 0,033 & 0,857 \\
& F3 & 0,337 & 1 & 0,337 & 0,020 & 0,888 \\
Grupo * Sexo & F4 & 97,068 & 1 & 97,068 & 0,417 & 0,519 \\
& F1 & 400,948 & 2 & 200,474 & 0,289 & 0,750 \\
& F2 & 8,880 & 2 & 4,440 & 0,464 & 0,630 \\
& F3 & 51,080 & 2 & 25,540 & 1,517 & 0,223 \\
& F4 & 4,799 & 2 & 2,399 & 0,010 & 0,990 \\
\hline
\end{tabular}


número de participantes por grupo mostrou-se mais equilibrado.

Os resultados dessa nova análise mostraram que a variável grupo influencia significativamente o resultado dos participantes no Fator 1 da criatividade (enriquecimento de idéias), a favor do grupo com inteligência acima da média. Novamente o sexo não exerceu influência sobre nenhum dos fatores criativos. Interessantemente a interação entre sexo e grupo no Fator 2 que havia sido apontada como significativa na análise anterior, não apresenta-se na presente análise.

De acordo com os resultados encontrados uma interessante discussão pode ser realizada. A primeira análise conduzida apontou diferenças marginalmente significativas na interação das variáveis sexo e grupo no fator emocional da criatividade, a favor das mulheres que apresentam alto nível de inteligência (percentil maior que 75 ), situação que não se mantêm quando o ponto de corte adotado utiliza como critério o percentil 66. Assim, o que se pode verificar é que, somente nos altos níveis de criatividade, as mulheres se diferenciam dos homens em relação aos aspectos emocionais da criatividade, apresentando de forma mais intensa e presente as habilidades que envolvem expor bem suas emoções e a utilização desse recurso como forma de compreender melhor a situação que lhes é apresentada como problema. Um bom resultado nesse fator caracteriza pessoas com bom desempenho nas habilidades emocionais, as quais normalmente não tem medo de sofrer críticas (Torrance \& Ball, 1990; Torrance \& Safter, 1999). Mesmo quando não obtêm o sucesso esperado, continuam insistindo em suas idéias, sem que isso o desmotive a continuar a busca pela solução (Wechsler, 2002). É nesse ponto que as pessoas criativas se diferenciam das demais, pois confiam em si mesmas e na sua capacidade de resolver problemas, ao contrário de muitas pessoas que desistem por medo de fracassar e medo do que os outros irão pensar. Encontram-se tão envolvidos e motivados que não se importam com os riscos que tem que correr, com o medo de serem criticados (Nakano et al., 2011). Isso porque as emoções têm sido vistas como facilitadoras dos processos de iluminação e inspiração ao permitirem soluções criativas para os proble- mas. Ela funcionaria como um efeito facilitador da criatividade (Nakano, 2012a).

Outra constatação interessante refere-se à influência do grupo no fator relacionado ao Enriquecimento de Idéias, a favor dos participantes que apresentam percentil acima de 66 no teste de inteligência. Assim podemos ver que aquelas pessoas que apresentam inteligência acima da média também tendem a apresentar alta habilidade em elaboração-perspectiva e a serem bastante persistentes em suas idéias. Concentram grande esforço no sentido de aperfeiçoar ou melhorar suas idéias, mesmo que elas já tenham sido tentadas antes. Apresentam empenho e dedicação necessária, possuindo ainda boa capacidade de planejamento e organização (Nakano, 2012b). Outra capacidade bem desenvolvida é a habilidade de ver as coisas sob diferentes perspectivas e pontos de vista, como uma forma de permitir a compreensão do problema dentro de um universo maior, através da inserção da solução dentro de um contexto (Nakano et al., 2011). Estas habilidades têm sido consideradas como importantes características apresentadas pelas pessoas criativas, sendo uma das grandes preditoras da realização criativa (Wechsler, 2002).

Outra importante observação refere-se ao fato de que quando o controle do nível de inteligência é realizado, as diferenças de sexo não mostram-se significativas, confirmando a idéia difundida na literatura acerca da androgenia psicológica, representada por autores como Aranha (1997), os quais negam a existência de distinção, entre os sexos, na criatividade. Para os autores desta posição teórica, os indivíduos criativos escapariam em certa medida ao rígido estereótipo dos papéis em função do gênero, o que seria explicado a partir da questão da androgenia psicológica, descrita como uma das características de personalidade presente nos individuos criativos (Montuori \& Purser, 1995). Ela se referiria à capacidade que um indivíduo tem para ser ao mesmo tempo, agressivo e protetor, sensível e rígido, dominante e submisso, de forma que, pessoas criativas se assemelhariam mais entre si do que em função do gênero.

Um indivíduo com essas características "duplica o repertório de reações e pode se relacionar com o mundo, partindo de um leque de possi- 
bilidades muito mais rico e variado" (Candeias, 2008, p. 53). Dessa maneira, De La Torre (2005) argumenta que as pessoas criativas fogem, facilmente, dos estereótipos de gênero. Enquanto as mulheres podem se manifestar dominantes e duras, menos submissas, os homens aparecem com maior grau de sensibilidade, não se agarrando aos papéis, culturalmente estabelecidos, o que acaba por gerar maior facilidade de adaptação.

Observações que têm sido feitas, por autores como Morais (2001), mostram que em relação à pesquisa da criatividade por sexo da amostra, tem sido observada uma diversidade de resultados, se forem tomadas investigações que envolvem duas ou três décadas,

assiste-se à afirmação da superioridade de cada um dos sexos, mas também, à negação de diferenças. A maioria, porém, implica a igualdade entre os sexos nesses formatos de realização criativa, não obstante se verificarem oscilações em função do conteúdo avaliado (Morais, 2001, p. 102).

Estas observações, não têm como objetivo afirmar, categoricamente, a superioridade criativa de um dos sexos, já que os estudos analisados apresentaram dados e conclusões baseadas em objetivos e amostras diferentes, entretanto os resultados da presente pesquisa mostraram que, quando se controla o nível de criatividade, as diferenças de sexo não se mostram significativas na amostra considerada.

Por fim com o objetivo de verificar a relação entre os dois construtos a correlação entre as duas medidas foi realizada, sendo os resultados apresentados na Tabela 4.

Tabela 4

Correlação de Pearson entre o TCFI e a BPRi

\begin{tabular}{|c|c|c|c|c|c|c|}
\hline & & Raciocinio total & RV & $\mathrm{RP}$ & RA & $\mathrm{RN}$ \\
\hline \multirow[t]{2}{*}{ F1 } & Correlação de Pearson & $0,230^{* *}$ & $0,266^{* *}$ & $0,171^{*}$ & $0,187^{*}$ & 0,090 \\
\hline & Sig. & 0,007 & 0,002 & 0,047 & 0,030 & 0,297 \\
\hline \multirow[t]{2}{*}{$\mathrm{F} 2$} & Correlação de Pearson & $0,179^{*}$ & 0,146 & 0,104 & 0,120 & 0,147 \\
\hline & Sig. & 0,038 & 0,090 & 0,229 & 0,165 & 0,090 \\
\hline \multirow[t]{2}{*}{ F3 } & Correlação de Pearson & 0,056 & 0,081 & $-0,011$ & 0,044 & 0,034 \\
\hline & Sig. & 0,519 & 0,348 & 0,900 & 0,615 & 0,693 \\
\hline \multirow[t]{2}{*}{$\mathrm{F} 4$} & Correlação de Pearson & 0,161 & $0,179^{*}$ & 0,169 & 0,096 & 0,076 \\
\hline & Sig. & 0,063 & 0,038 & 0,050 & 0,270 & 0,381 \\
\hline \multirow[t]{2}{*}{ Criatividade total } & Correlação de Pearson & $0,228^{* *}$ & $0,258^{* *}$ & $0,181^{*}$ & $0,170^{*}$ & 0,101 \\
\hline & Sig. & 0,008 & 0,002 & 0,036 & 0,048 & 0,242 \\
\hline
\end{tabular}

${ }^{*} \mathrm{p} \leq 0,05 ; * \mathrm{*} \leq 0,01$.

Os resultados demonstram uma correlação baixa $(r=0,22, p \leq 0,008)$ entre as medidas de criatividade e inteligência. Se analisadas cada uma das provas de raciocínio poderemos ver que o raciocínio verbal é o que apresenta maior número de correlações significativas com a criatividade (Fator 1, Fator 4 e criatividade total). As provas de raciocínio prático e abstrato também apresentam correlações significativas com o Fator 1 e criatividade total, devendo ser destacado o fato de que a prova de raciocínio numérico não apresentou correlação significativa com nenhuma das medidas de criatividade.

A constatação de existência de correlação concorda com estudos encontrados na literatura, os quais têm demonstrado que inteligência e criatividade podem ser considerados construtos diferentes, porém relacionados entre si. Nesse sentido a literatura tem apresentado estudos que apresentaram valores mais de correlação próximos ao que foi encontrado, podendo ser citada a pesquisa desenvolvida por Rindermann e Neu- 
bauer (2004) que, fazendo uso de dois testes de inteligência (Raven e Kognitiver FahigkeitsTest) e dois testes de criatividade (Verbaler Kreativitats-Test e Verwendungs-Test) em 271 estudantes do Ensino Médio relataram correlações de 0,14 entre as medidas de criatividade e inteligência. Outros estudos, tais como o de Elisondo e Donolo (2010) ao investigar grupos diferentes (alunos de nível secundário e universitários em um total de 962 participantes), utilizando-se a prova CREA e o Test Elemental de Inteligencia para avaliar alunos do ensino médio e Matrizes Progressivas de Raven para os universitários, encontraram valores de correlação de 0,16 para os estudantes do ensino médio e de 0,14 para os estudantes do ensino superior.

A partir da aplicação de diversos instrumentos em universitários, para avaliação da criatividade (Divergent Thinking Fluency, Rated Creativity, Biographical Inventory of Creative Behaviours, Self-rating of creativity) e para avaliação da inteligência (Raven's Advanced Progressive Matrices, General Knowledge Questionnaire), Batey, Furnham e Safiullina (2010) encontraram correlações de 0,29 entre a criatividade total e o resultado no Raven. Estudo brasileiro desenvolvido por Wechsler, Nunes, Schelini, Ferreira e Pereira (2010) junto a 172 estudantes com idades entre 7 e 18 anos a partir do uso da Bateria Woodcock- Johnson III e os Testes de Pensamento Criativo de Torrance também apontou a existência de correlações significativas, na amostra total, entre inteligência e o índice criativo figural I, que compreende os componentes cognitivos da criatividade figural, na ordem de 0,22 e de 0,20 para o índice criativo figural II, que compreende os componentes cognitivos e emocionais, fato que não ocorreu em relação à criatividade verbal.

Uma revisão mostra que resultados moderados de correlação também são apontados em diversas pesquisas. Runko e Mraz (1992), por exemplo, relataram correlações de 0,58 entre criatividade e inteligência em uma amostra composta por 30 estudantes universitários, Lundsteen (1966) apontou uma relação na ordem de 0,40 encontrada por Wallach e Kogan em um estudo envolvendo estudantes de três grupos: um com alta criatividade e alta inteligência, outro grupo com alta medida em somente um dos construtos e outro com baixas medidas em ambos. Também tendo como foco a investigação das diferenças entre grupos, Preckel et al. (2006) em uma amostra de 1328 estudantes de $4^{\mathrm{a}}$ a $7^{\mathrm{a}}$ séries, classificados em três grupos: baixa, media e alta inteligência (com este ultimo grupo fazendo parte de uma escola especializada em superdotados) fizeram uso do Berlin Structure-of-Intelligence-test (BIS-HB), sendo que os resultados apontaram que, para a amostra total, inteligência correlacionou-se com criatividade $(r=0,54)$, com criatividade verbal $(r=0,51)$, criatividade figural $(r=0,36)$ e criatividade numérica $(r=0,38)$.

Correlação mediana também foi encontrada em um estudo brasileiro, conduzido por Barros, Primi, Miguel, Almeida e Oliveira (2010), no qual uma medida de criatividade (Teste de Criação de Metáforas) apresentou correlação significativa com duas medidas de raciocínio, sendo de 0,31 com raciocínio abstrato e de 0,48 com raciocínio verbal. Estudo um pouco diferente foi conduzido por Silvia (2008) junto a 226 estudantes universitários que completaram uma série de testes de criatividade (usos incomuns) e inteligência (raciocínio fluído, fluência verbal e geração de estratégias). O autor buscou identificar o efeito da inteligência na criatividade, tendo encontrado um fator de primeira ordem cujo efeito médio-grande na criatividade $(0,43)$, sugerindo que um fator de inteligência geral mostrou-se mais relevante para a criatividade do que fatores específicos.

Por outro lado, Aguirre e Conners (2010) relatam ausência de relação significativa em crianças pré-escolares após resposta a dois testes de criatividade (Thinking Creatively in Action and Movement e Multidimensional Stimulus Fluency Measure) e um de inteligência (Stanford Binet $V$ Abbreviated). Os autores concluíram que o número de participantes $(n=27)$ era adequado para detectar um poder de correlação grande, de forma a se afirmar que se a relação existe, ela não deve ser grande, embora argumentem que os resultados não podem ser generalizados devido ao número limitado de participantes.

Por fim torna-se importante destacar que, dependendo de como os construtos são medidos, do instrumento utilizado, da teoria que os em- 
basa, das diferenças metodológicas e da amostra estudada (que varia em função da idade, habilidade e nível educacional), os resultados têm apresentado amplas variações (Preckel et al., 2006). Embora a maior parte destes tenha concluído acerca da existência de alguma relação entre criatividade e inteligência, com a amplitude desta relação variando entre baixa e moderada, as diferenças relatadas acabam dificultando o consenso em relação a esta questão.

Estudos envolvendo o instrumento de criatividade utilizado apontam claramente para a influência desses fatores (amostra e instrumento utilizado) nos resultados encontrados. Pesquisa fazendo uso do mesmo instrumental, também com a finalidade de comparar criatividade e inteligência, indicaram resultados bastante diferentes, dependendo do instrumento de inteligência utilizado. Quando esse construto foi avaliado a partir de um instrumento de desenvolvimento cognitivo, Desenho da Figura Humana, a correlação entre os desempenhos mostrou-se uma amplitude moderada $(r=0,47)$, devendo-se atentar ao fato de que tal valor pode ter sido influenciado pela similaridade da tarefa a ser realizada nos dois instrumentos (desenhos), visto que outros estudos comparando o teste de criatividade com outras medidas de inteligência apresentaram ausência de correlação significativa, tanto com a versão padrão (Chiodi, Nakano, \& Wechsler, 2011a) quanto ampliada da Bateria Woodcock-Johnsson III (Chiodi, Nakano, \& Wechsler, 2011b).

\section{Considerações Finais}

Espera-se que os resultados encontrados na pesquisa possam enriquecer as discussões, bastante presentes na literatura, sobre a relação entre criatividade e inteligência. Sabe-se que pesquisas brasileiras sobre a temática ainda são escassas, principalmente aquelas que visam identificar essas habilidades em crianças e adolescentes.

Novos estudos com diversificação da amostra e dos instrumentos utilizados são recomendados, visto que, conforme apontado, pesquisas fazendo uso do mesmo instrumental de criati- vidade têm apontado magnitudes diferentes de relação com a inteligência, dependendo do teste utilizado, de seu embasamento teórico e tipo de atividade. Espera-se que o aprofundamento desses estudos permitam uma compreensão mais efetiva acerca da relação entre os dois construtos, assim como avanços na investigação por meio de novos estudos e busca de respostas para essa questão.

\section{Referências}

Ackerman, P. L., Bowen, K. R., Beier, M. E., \& Kanfer, R. (2001). Determinants of individual differences and gender differences in knowledge. Journal of Educational Psychology, 93(4), 797-825.

Aguirre, K., \& Conners, F. (2010). Creativity and inteliigence in preschoolers: Preliminary findings. The University of Alabama McNair Journal. Retrieved May 05, 2011, from http://graduate. ua.edu/mcnair/journals/2010/Aguirre.pdf

Albert, R. S., \& Runko, M. A. (1999). A history of research on creativity. In R. J. Sternberg (Ed.), Handbook of creativity (pp. 16-31). Cambridge, UK: Cambridge University Press.

Alencar, E. M. L. S. (1975). Efeitos de um programa de treinamento de criatividade em alunos de $4^{\mathrm{a}}$ e $5^{\text {a }}$ séries. Arquivos Brasileiros de Psicologia Aplicada, 27(4), 3-15.

Alencar, E. M. L. S. (1997). O estímulo à criatividade no contexto universitário. Psicologia Escolar e Educacional, 1(2-3), 29-37.

Almeida, L. S. (1988). O impacto das experiências educativas na diferenciação cognitiva dos alunos: Análise dos resultados em provas de raciocínio diferencial. Revista Portuguesa de Psicologia, 24, 131-157.

Almeida, L. S., \& Campos, B. P. (1985). Raciocínio diferencial de jovens: Experiências escolares e diferenças de sexo. Cadernos de Consulta Psicológica, 1, 41-51.

Almeida, L. S., Lemos, G. C., \& Primi, R. (2011). Recensão critica: Bateria de Provas de Raciocínio (BPR). In C. Machado, M. Gonçalves, L. S. Almeida, \& M. R. Simões (Orgs.), Instrumentos e contextos de avaliação psicológica (pp. 285311). Coimbra, Portugal: Almedina. 
Almeida, L. S., \& Primi, R. (2000). Bateria de Provas de Raciocínio (BPR-5). São Paulo, SP: Casa do Psicólogo.

Aranha, M. A. R. C. (1997). Creativity in students and its relation to intelligence and peer perception. Revista Interamericana de Psicologia, 31(2), 309-313.

Back, R., \& Dana, R. H. (1977). Examiner sex bias and Wechsler Intelligence Scale for children scores. Journal of Consulting and Clinical Psychology, 45(3), 500.

Baer, J. (1999). Gender differences. In M. A. Runko \& S. R. Pritzker (Eds.), Encyclopedia of creativity (Vol. 1, pp. 753-758). San Diego, CA: Academic Press.

Barros, D. P., Primi, R., Miguel, F. K., Almeida, L., \& Oliveira, E. P. (2010). Metaphor creation: A measure of creativity or intelligence?. European Journal of Education and Psychology, 3(1), 103-115.

Batey, M., Furnham, A., \& Safiullina, X. (2010). Intelligence, general knowledge and personality. Learning and Individual Differences, 20, 532535.

Becker, M. (1995). Ninetheenth century foundations of creativity research. Creativity Research Journal, 8, 219-229.

Bornstein, R. A. (1984). Unilateral lesions and the Wechsler Adult Intelligence Scale-Revised: No sex differences. Journal of Consulting and Clinical Psychology, 52(4), 604-608.

Camarata, S., \& Woodcock, R. (2006). Sex differences in processing speed: Developmental effects on males and females. Intelligence, 34, 231-252.

Candeias, A. A. (2008). Criatividade: Perspectiva integrativa sobre o conceito e a sua avaliação. In M. F. Morais \& S. Bahia (Orgs.), Criatividade: Conceito, necessidades e intervenção (pp. 4164). Braga, Portugal: Psiquilíbrios.

Chiodi, M. G., Nakano, T. C., \& Wechsler, S. M. (2011a, jun.). Estudo de correlação entre criatividade e habilidades intelectuais. Trabalho apresentado no I Congresso Internacional de Criatividade. Inovação, Manaus, AM, Brasil.

Chiodi, M. G., Nakano, T. C., \& Wechsler, S. M. (2011b, jun.). Inteligência e criatividade: Um estudo correlacional. Trabalho apresentado no I Congresso Internacional de Criatividade. Inovação, Manaus, AM, Brasil.
Cruz, M. B. Z. (2008). Estudo de validade e precisão da bateria de provas de raciocínio infantil - BPR-5I (Dissertação de mestrado em Psicologia, Universidade de São Francisco, Itatiba, SP, Brasil).

De La Torre, S. (2005). Dialogando com a criatividade. São Paulo, SP: Madras.

Doherty, S. J., Kovas, Y., \& Plomin, R. (2011). Gene-environment interaction in the etiiology of mathematical ability using SNP sets. Behavior Genetics, 41, 141-154.

Dudek, S. Z., Strobel, M. G., \& Runko, M. A. (1993). Cumulative and proximal influences on the social enviroment and children's creative potential. Journal of Genetic Psychology, 154(4), 487-500.

Edwards, M. P., \& Tyler, L. E. (1965). Intelligence, creativity and achievement in nonselective public junior high school. Journal of Educational Psychology, 56(2), 96-99.

Elisondo, R. C., \& Donolo, D. S. (2010). ¿Creatividad o inteligencia? That is not the question. Anales de Psicología, 26(2), 220-225.

Getzels, J. W., \& Jackson, P. W. (1962). Creativity and intelligence: Explorations with gifted students. New York: John Wiley \& Sons.

Halari, R., Hines, M., Kumari, V., Mehrotra, R., Wheeler, M., \& Ng, V. (2005). Sex differences and individual differences in cognitive performance and their relationship to endogenous gonadal hormones and gonadotropins. Behavioral Neuroscience, 119(1), 104-117.

Hamilton, J., \& McKenna, B. (1965). Sex differences in the Wechsler Intelligence Tests. The Canadian Psychologist, 6(4), 353-357.

Hattie, J., \& Rogers, H. J. (1986). Factor models for assessing the relation between creativity and intelligence. Journal of Educational Psychology, $78(6), 482-485$.

Jensen, A. R. (1998). The g factor. Westport, CT: Prager.

Keith, T. Z., Reynolds, M. R., Patel, P. G., \& Ridley, K. P. (2008). Sex differences in latent cognitive abilities ages 6 to 59: Evidence from the Woodcock-Johnson III tests of cognitive abilities. Intelligence, 36, 502-525.

Kim, K. H. (2005). Can only intelligent people be creative? Journal of Secondary Gifted Education, 16, 57-66. 
Kim, K. H., Cramond, B., \& Bandalos, D. L. (2006). The latent structure and measurement invariance of scores on the Torrance tests of Creative Thinking-Figural. Educational and Psychological Measurement, 66(3), 459-477.

Kimura, D. (1987). Are men's and women's brains really different? Canadian Psychology, 28(2), 133-147.

Kneller, G. F. (1971). Arte e ciência da criatividade. São Paulo, SP: Ibrasa.

Kramer, J. H., Kaplan, E., Delis, D. C., O’Donnell, L., \& Prifitera, A. (1997). Developmental sex differences in verbal learning. Neuropsychology, 11(4), 577-584.

Lemos, G., Almeida, L. S., Guisande, M. A., Primi, R., Martinho, G., \& Fortes, I. (2010). Inteligência e rendimento escolar: Contingências de relacionamento menos óbvio no final da adolescência. Revista Galego-Portuguesa de Psicoloxía e Educación, 18, 1138-1663.

Lubart, T. (2007). Psicologia da criatividade. Porto Alegre, RS: ArtMed.

Lundsteen, S. W. (1966). Book reviews: Modes of thinking in young children: A study of creativity-intelligence distinction by Michael A. Wallach and Nathan Kogan. Educational and Psychological Measurement, 26, 523-527.

Martinez, O. L., \& Lozano, J. N. (2010). Rasgos de personalidad y desarrollo de la creatividad. Anales de Psicología, 26, 151-158.

Montuori, A., \& Purser, R. E. (1995). Deconstructing the lone genius myth: Toward a contextual view of creativity. Journal of Humanistic Pyschology, 35(3), 69-112.

Morais, M. F. (2001). Criatividade como (re)conciliação: Indivíduo, cultura e acaso. Psicologia: Teoria, Investigação e Prática, 1, 97-121.

Mouchiroud, C., \& Lubart, T. (2002). Social creativity: A cross-sectional study of 6 to 11 year-old children. International Journal of Behavioral Development, 26(1), 60-69.

Nakano, T. C. (2012a). Teste de Criatividade Figural Infantil. In C. S. Hutz (Org.), Avanços em Avaliação Psicológica e Neuropsicológica de crianças e adolescentes II (pp. 425-451). São Paulo, SP: Casa do Psicólogo.

Nakano, T. C. (2012b). O desenho na expressão criativa: Teste de Criatividade Figural Infantil. In S. M. Wechsler \& T. C. Nakano (Orgs.), $O$ desenho infantil: Forma de expressão cognitiva, criativa e emocional (pp. 67-95). São Paulo, SP: Casa do Psicólogo.

Nakano, T. C., \& Primi, R. (2012). A estrutura fatorial do Teste de Criatividade Figural Infantil. Psicologia: Teoria e Pesquisa, 28(3), 91-100.

Nakano, T. C., \& Wechsler, S. M. (2006). Teste Brasileiro de Criatividade Figural: Proposta de instrumento. Interamerican Journal of Psychology, 40(1), 103-110.

Nakano, T. C., Wechsler, S. M., \& Primi, R. (2011). Teste de Criatividade Figural Infantil. São Paulo, SP: Vetor.

Nisbett, R. E., Aronson, J., Blair, C., Dickens, W., Flynn, J., Halpern, D. F., \& Turkheimer, E. (2012). Intelligence: Findings and theoretical developments. American Psychologist, 67(2), 130-159.

Nogueira, S. M., \& Pereira, M. (2008). Estratégias promotoras da criatividade. In M. F. Morais \& S. Bahia (Orgs.), Criatividade: Conceito, necessidades e intervenção (pp. 253-278). Braga, Portugal: Psiquilibrios.

Osborn, A. F. (1975). O poder criador da mente: Princípios e processos do pensamento criador e do brainstorming. São Paulo, SP: Ibrasa.

Pereira, M. (2000). Sobredotação: A pluralidade do conceito. Sobredotação, 1(1-2), 147-178.

Preckel, F., Holling, H., \& Wiese, M. (2006). Relationship of intelligence and creativity in gifted and non-gifted students: An investigation of threshold theory. Personality and Individual Differences, 40, 159-170.

Primi, R., \& Almeida, L. S. (n.d.). Bateria de Provas de Raciocínio Infantil. Manuscrito não publicado.

Reese, H. W., Lee, L. J., Cohen, S. H., \& Puckett, J. M., Jr. (2001). Effects of intellectual variables, age, gender on divergent thinking in adulthood. International Journal of Behavioral Development, 25(6), 491-500.

Reynolds, M. R., Keith, T. Z., Ridley, K. P., \& Patel, P. G. (2008). Sex differences in latent general and broad cognitive abilities for children and youth: Evidence from higher order MG-MACS and MIMIC models. Intelligence, 36, 236-260.

Rindermann, H., \& Neubauer, A. C. (2004). Processing speed, intelligence, creativity, and school performance: Testing of causal hypotheses using structural equation models. Intelligence, 32, 573-589. 
Rodrigues, C. J. S., \& Alencar, E. M. L. S. (1983). Um estudo do pensamento criativo em alunos do $1^{\circ}$ grau. Arquivos Brasileiros de Psicologia, 35(1), 113-122.

Runko, M. A., \& Mraz, W. (1992). Scoring divergent thinking tests using total ideational output and a creativity index. Educational and Psychological Measurement, 52, 213-221.

Russo, C. F. (2004). Comparative study of creativity and cognitive-solving strategies of high-IQ and average students. Gifted Child Quarterly, 48(3), $179-190$

Sawyer, R. K. (2006). Explaining creativity. New York: Oxford University Press.

Shaffer, D. R. (2005). Psicologia do desenvolvimento. São Paulo, SP: Thomson Learning.

Silvia, P. J. (2008). Another look at creativity and intelligence: Exploring higher-order models and probable confounds. Personality and Individual Differences, 44, 1012-1021.

Sternberg, R. J. (1984). What should intelligence tests test? Implications of a triarchic theory of intelligence for intelligence testing. Educational Researcher, 13, 5-15.

Sternberg, R. J. (2000). Handbook of intelligence. New York: Cambridge University Press.

Sternberg, R. J. (2001). What is the common thread of creativity? Its dialectical relation to intelligence and wisdom. American Psychological Association, 56(4), 360-362.

Sternberg, R. J., \& O’Hara, L. A. (2000). Intelligence and creativity. In R. J. Sternberg (Ed.), Handbook of intelligence (pp. 611-630). New York: Cambridge University Press.

Torrance, E. P., \& Ball, O. E. (1990). Streamlined Scoring and Interpretation Guide and Norms Manual Verbal and Figural Form B. Bensenville, IL: Scholastic Testing Service.

Torrance, E. P., \& Safter, H. T. (1999). Making the creative leap beyond. Buffalo, NY: Creative Education Foundation.

van der Sluis, S., Derom, C., Thiery, E., Bartels, M. Polderman, T., Verhulst, F. C., ...Posthuma, D. (2008). Sex differences on the WISC-R in Belgium and Netherlands. Intelligence, 36, 48-67.

Virgolim, A. M. R., \& Alencar, E. M. L. S. (1993). Habilidades de pensamento criativo entre alunos de escolas abertas, intermediárias e tradicionais. Psicologia: Teoria e Pesquisa, 9(3), 601-610.
Wechsler, S. M. (1987). Efeitos do treinamento em criatividade em crianças bem dotadas e regulares. Arquivos Brasileiro de Psicologia, 4, 95110.

Wechsler, S. M. (2002). Avaliação da criatividade por figuras e palavras: Testes de Torrance Versão brasileira. Campinas, SP: Lamp.

Wechsler, S. M., \& Nakano, T. C. (2002). Caminhos para a avaliação da criatividade: Perspectiva brasileira. In R. Primi (Org.), Temas em Avaliação Psicológica. (pp. 103-115). São Paulo, SP: Instituto Brasileiro de Avaliação Psicológica.

Wechsler, S. M., \& Nakano, T. C. (2003). Produção brasileira em criatividade: $\mathrm{O}$ estado da arte. $E s$ critos sobre Educação, 2(2), 43-50.

Wechsler, S. M., \& Nakano, T. C. (2006). O percurso da criatividade figural do Ensino Médio ao Ensino Superior. Boletim de Psicologia, 56(125), 205-219.

Wechsler, S. M., Nunes, M. F. O., Schelini, P. W., Ferreira, A. A., \& Pereira, D. A. P. (2010). Criatividade e inteligência: Analisando semelhanças e discrepâncias no desenvolvimento. Estudos de Psicologia (Natal), 5(3), 243-250.

Wechsler, S. M., \& Richmond, B. (1984). Influências da dotação intelectual e criativa no ajustamento em sala de aula. Arquivos Brasileiros de Psicologia, 36(2), 138-147.

Zanella, A. V., \& Titon, A. P. (2005). Análise da produção científica sobre criatividade em programas brasileiros de pós-graduação em psicologia (1994-2001). Psicologia em Estudo, 10(2), 305-316. 